\title{
French Language
}

National Cancer Institute

\section{Source}

National Cancer Institute. French Language. NCI Thesaurus. Code C123785.

A romance language spoken as the official language in France and 28 other countries. 